\title{
ANÁLISE MULTICRITÉRIO DA ESCOLHA DE UM NOVO CURSO EM UMA INSTITUIÇÃO DE ENSINO TECNOLÓGICO ${ }^{1}$
}

\author{
Edilacy da Silva Sampaio ${ }^{\text {ab }}$, Valério A. P. Salomon ${ }^{a^{*}}$ \\ ${ }^{a}$ Departamento de Produção, Faculdade de Engenharia de Guaratinguetá \\ Universidade Estadual Paulista Júlio de Mesquita Filho - UNESP, Guaratinguetá-SP, Brasil \\ ${ }^{\mathrm{b}}$ Instituto Federal de Educação, Ciência e Tecnologia de Roraima - IFRR \\ Campus Novo Paraíso-RR, Brasil
}

Recebido 23/10/2018, aceito 23/06/2019

\begin{abstract}
RESUMO
Uma das primeiras decisões tomadas pela administração de uma instituição de ensino é a escolha dos cursos a serem oferecidos. Este artigo apresenta um estudo de caso sobre a escolha de um novo curso por uma instituição de ensino tecnológico na Região Norte do Brasil. $\mathrm{O}$ objetivo geral do estudo é resolver um problema de decisão discreto com a análise multicritério. AHP (Analytic hierarchy process) é o método de análise multicritério aplicado no caso. A aplicação do método AHP gerou um resultado conclusivo: Bacharelado em Zootecnia é o curso de nível superior indicado para a instituição estudada.
\end{abstract}

Palavras-chave: Analytic hierarchy process, Decisão discreta, Educação tecnológica.

\begin{abstract}
One of the first decisions made by managers for educational institution is the choice of the courses to be offered. This article presents a case study on the choice of a new course for a technological education institution in North Brazil. The objective of the study is to solve a problem of discrete decision with multi-criteria analysis. The multi-criteria analysis method applied in the case is AHP (Analytic hierarchy process). AHP application resulted in Bachelor of Animal Sciences as the new course for the institution.
\end{abstract}

Keywords: Analytic hierarchy process, Discrete decision, Technology education.

*Autor para correspondência. E-mail: valerio.salomon@unesp.br

DOI: $10.4322 /$ PODes.2019.004

${ }^{I}$ Todos os autores assumem a responsabilidade pelo conteúdo do artigo. 


\section{Introdução}

A autonomia universitária, prevista em lei, confere às instituições de ensino autoridade para escolher os cursos que desejam ofertar (Ranieri, 2015). Assim, uma das primeiras decisões tomadas pela administração da instituição de ensino é a escolha dos cursos a serem oferecidos. Como os recursos das instituições de ensino são limitados, o conjunto de cursos é finito. Uma instituição de ensino pode oferecer cursos em diversas áreas do conhecimento, ou em apenas uma, como, por exemplo, cursos na área de ciências da saúde (Costa et al., 2014).

Com o passar dos anos, podem surgir necessidades de mudanças nos cursos, incluindo alterações nos cursos atuais, exclusão e inclusão de novos cursos. Esta necessidade gera um "problema de decisão discreto" (Ishizaka e Nemery, 2013), ou seja, um problema com um número finito de soluções alternativas. Este problema de decisão pode ainda ser classificado como um "problema de escolha" ou como um "problema de ordenação", dependendo do número de soluções viáveis consideradas (Roy, 1981). Por exemplo, se houverem recursos para a implantação de um único curso, trata-se de um problema de escolha. Neste caso, quando apenas uma alternativa é escolhida, a segunda melhor alternativa é preterida, igualmente à pior alternativa. Ou seja, não importa a ordem das alternativas subsequentes. Por outro lado, se houverem recursos para a implantação de mais de um curso, trata-se de um problema de ordenação. Assim, a segunda melhor pode ser escolhida, num segundo momento; e as demais alternativas, em momentos seguintes, respeitando-se uma ordem.

Problemas de decisão discretos, como os problemas de escolha ou de ordenação, têm sido solucionados com métodos de análise multicritério (Steuer et al., 2017). Há aplicações bemsucedidas em diversas áreas do conhecimento, com destaque para Administração e Engenharia (Zopounidis e Pardalos, 2010). Mas, a gestão do ensino superior ou tecnológico é, relativamente, menos estudada (Khan et al., 2018). Assim, o presente artigo contribui para a literatura da análise multicritério apresentando uma aplicação prática em uma área não usual. AHP (Analytic hierarchy process) é o método de análise multicritério aplicado no artigo. A principal razão para escolha do método AHP é que se trata de um método consolidado de análise multicritério, conforme Seção 2.1. A escolha de um método de análise multicritério pode ser uma questão de fato ou uma questão de opinião (Salomon et al., 2013). Alguns autores defendem que um tipo de método é mais adequado para um tipo de problema (Roy, 1981; Ishizaka e Nemery, 2013). Adaptações ou variações em métodos originais podem ser necessárias em situações particulares. O escopo deste artigo não é a proposta de um novo método. O escopo do artigo é a aplicação do AHP em uma situação não corriqueira. Portanto, este artigo busca contribuições práticas, ou seja, contribuições de aplicação do AHP, o que justifica publicação em periódico científico, "apresentando um problema decisório ainda não solucionado pelo método com um toque de inovação" (Mu, 2017).

Este artigo apresenta um estudo de caso sobre a escolha de um novo curso por instituição de ensino tecnológico na Região Norte do Brasil. O objetivo geral do estudo é resolver o problema de decisão discreto com a análise multicritério. Objetivos específicos incluem identificar critérios para a escolha do curso e analisar a aplicação de um método de análise multicritério quanto á eficácia e eficiência da aplicação. Portanto, o artigo apresenta uma contribuição prática ao apresentar a aplicação do AHP em um problema de decisão não usual, relacionado com a gestão do ensino tecnológico. A aplicação do Modelo BOCR (BenefitsOpportunities-Costs-Risks) e da síntese ideal, ao invés da síntese normal, ampliam a originalidade do artigo.

O conteúdo deste artigo adota a Estrutura IMRAD, abreviação para Introduction, Methods, Results And Discussion (Williams, 2018). Assim, após esta seção de Introdução, na Seção 2, apresenta-se a metodologia da pesquisa, detalhando-se os passos de aplicação do AHP e conceitos como o Modelo BOCR; na Seção 3, os resultados da aplicação do AHP no caso real são analisados; finalmente, na Seção 4, discutem-se as conclusões do artigo. 


\section{Metodologia}

\subsection{Análise Multicritério}

Diversos métodos e técnicas de análise multicritério (Ishizaka e Nemery, 2013) têm sido desenvolvidos, desde a década de 1960. Os métodos são geralmentes referenciados por siglas como, por exemplo:

- AHP (Analytic hierarchy process);

- DEA (Data envelopment analysis);

- ELECTRE (Elimination et choix traduisant la réalité);

- MAUT (Multi-attributte utility theory);

- TOPSIS (Technique for the order of prioritisation by similarity to ideal solution).

Basicamente, a modelagem matemática da análise multicritério ocorre em três passos principais: Estruturação, Medição e Síntese. Na Estruturação, definem-se o objetivo da análise, os critérios e as alternativas. Na Medição, estabelecem-se pesos para os critérios e pesos para as alternativas de acordo com cada critério (pesos locais). Na Síntese, obtém-se pesos globais para as alternativas (agregando os pesos locais). Caso os resultados não sejam considerados satisfatórios, os passos anteriores podem ser revisados (Figura 1).

Figura 1: Modelagem matemática da análise multicritério.

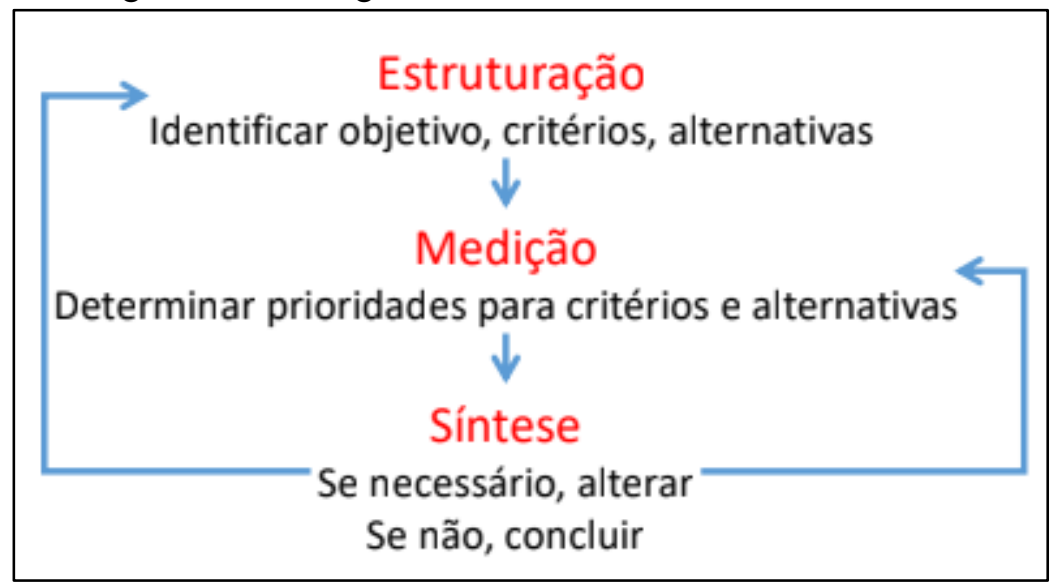

Fonte: Elaborada pelos autores.

O que torna um método de análise multicritério diferente do outro é a maneira com que os passos apresentados na Figura 1 são executados. A Matriz de Decisão $D$ (Equação 1) é a ferramenta principal utilizada na aplicação de diversos métodos. Há diferença de nomenclatura entre os métodos: dij pode representar o peso, pertinência, possibilidade ou prioridade da Alternativa $i$ com relação ao Critério $j$.

$$
D=\left\{\begin{array}{cccc}
d_{11} & d_{12} & \ldots & d_{1 n} \\
d_{21} & d_{22} & \ldots & d_{2 n} \\
\ldots & \ldots & & \ldots \\
d_{m 1} & d_{m 2} & \ldots & d_{m n}
\end{array}\right\}
$$

Apesar da existência de diversos métodos para análise multicritério, observa-se que há uma concentração de estudos nos métodos AHP e DEA (Wallenius et al., 2008; Khan et al., 2018). Portanto, AHP é um método consolidado para a análise multicritério. Esta é uma das razões para escolha deste método no caso apresentado neste artigo. Ou seja, como é a primeira aplicação de um método de análise multicritério na instituição, optou-se por um método consolidado buscando-se mitigar riscos de aplicação equivocada. Outra razão para a escolha do 
AHP deve-se pela característica esperada do problema discreto: poucas alternativas devem ser analisadas. Para aplicação do método DEA, necessita de que o número de alternativas seja maior que o dobro do número de critérios (Silva et al., 2014). Quando o número de alternativas não obedece à esta regra, a aplicação do DEA pode não discriminar as alternativas.

\subsection{Analytic Hierarchy Process}

No método AHP original (Saaty, 1980), os passos da modelagem matemática (Figura 1) são executados com adoção de: Estruturas hierárquicas (Figura 2), no passo de Estruturação; Escala Saaty (Tabela 1), autovetor direito $w$ e autovalor máximo $\lambda_{\max }$ de matrizes de comparações aos pares $A$, na Medição; Síntese Normal dos pesos, ou seja, o vetor dos pesos é obtido com a normalização do autovetor, de modo que a soma dos seus componentes seja igual a 1 .

Em uma estrutura hierárquica (Figura 2), os objetos da análise multicritério são dispostos em níveis hierárquicos: no nível mais alto da hierarquia está o objetivo da análise; no nível intermediário, os critérios; no nível mais baixo, as alternativas. Modelos complexos de análise multicritério são representados por estruturas hierárquicas com mais de três níveis, por exemplo, incluindo subcritérios ou alternativas secundárias.

Figura 2: Estrutura hierárquica.

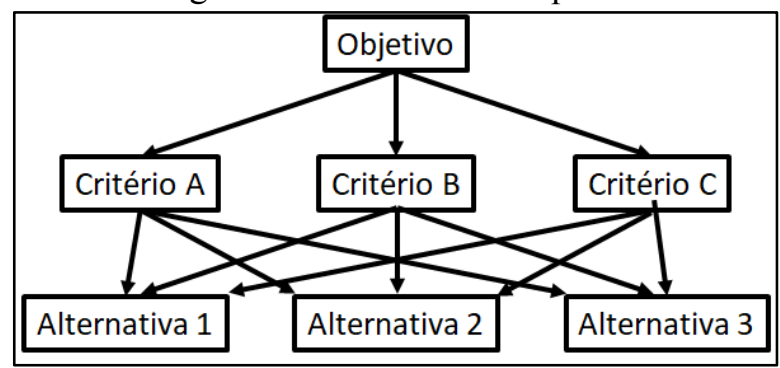

Fonte: Elaborada pelos autores.

Modelo BOCR (Benefits-Opportunities-Costs-Risks) é frequentemente utilizado para a identificação de critérios (Saaty e Islam, 2015). Os critérios Benefícios e Custos representam os aspectos favor'aveis e desfavor'aveis que certamente ocorrem com a escolha de uma alternativa, enquanto Oportunidades e Riscos representam os aspectos incertos (Tramarico et al., 2012). Com o uso do Modelo BOCR pretende-se identificar crit'erios mutualmente excludentes e coletivamente exaustivos.

No AHP, os pesos dos critérios e das alternativas são denominados "prioridades". As prioridades são obtidas com o autovetor direito $\boldsymbol{w}$ (de dimensão $n \times 1$ ) da matriz de comparações aos pares $\boldsymbol{A}$ (de dimensão $n \times n$ ), conforme a Equação 2, onde $\lambda_{\max }$ é o autovalor máximo:

$$
\boldsymbol{A} \boldsymbol{w}=\lambda_{\max } \boldsymbol{w}
$$

As comparações, geralmente, são realizadas baseando-se na Escala Saaty (Tabela 1), ou Escala Fundamental de Números Absolutos (Saaty, 2015).

Tabela 1: Escala de Saaty.

\begin{tabular}{ccc}
\hline Intensidade & Definição & Explicação \\
\hline 1 & Importância igual & Dois objetos contribuem igualmente para o nível superior \\
3 & Importância & Julgamento levemente mais favorável para um objeto \\
& moderada & \\
5 & Importância forte & Julgamento fortemente mais favorável para um objeto \\
7 & Importância muito & O domínio de um objeto é praticamente evidente \\
\hline
\end{tabular}


forte

9

Importância

Há evidência favorável para um objeto na mais alta ordem extrema possível de afirmação

Fonte: adaptada de Saaty (2015).

A Tabela 2 apresenta uma matriz de comparações aos pares entre três critérios (X, Y e Z) e o seu vetor de prioridades. Nota-se que $a_{i i}=1$ e $a_{i j}=1 / a_{j i}$. Nota-se ainda que o vetor de prioridades dos pesos foi normalizado.

Tabela 2: Comparações e prioridades dos critérios X, Y e Z.

\begin{tabular}{ccccc}
\hline Critério & $\mathrm{X}$ & $\mathrm{Y}$ & $\mathrm{Z}$ & Prioridade \\
\hline $\mathrm{X}$ & 1 & $1 / 3$ & 3 & $24 \%$ \\
$\mathrm{Y}$ & 3 & 1 & 5 & $65 \%$ \\
$\mathrm{Z}$ & $1 / 3$ & $1 / 5$ & 1 & $10 \%$ \\
\hline \multicolumn{5}{c}{ Fonte: Elaborada pelos autores. }
\end{tabular}

O autovalor máximo $\lambda_{\max }$ é uma medida da consistência da matriz $\boldsymbol{A}$. Ou seja, se todas as comparações forem coerentes entre si, então, a Equação 3 se verifica para todos os $i=1,2,3 \ldots n$ e $j=1,2,3 \ldots n$. Neste caso, $\lambda_{\max }=n$ (Saaty, 1980).

$$
a_{i j}=\frac{w_{i}}{w_{j}}
$$

$\mathrm{O}$ índice de consistência $C I$ (consistency index), compara o afastamento entre $\lambda_{\max }$ e $n$ com os graus de liberdade, $n-1$, conforme a Equação 4:

$$
C I=\frac{\lambda_{\max }-n}{n-1}
$$

A razão de consistência $C R$ (consistency ratio) é uma medida melhor da consistência de uma matriz de comparações, pois considera um índice aleatório, RI (random index), conforme a Equação 5:

$$
C R=\frac{C I}{R I}
$$

Usualmente, considera-se a inconsistência da matriz de comparações aceitável para valores de $C R \leq 0,10$ (Saaty e Islam, 2015). Para a matriz de comparações apresentada na Tabela 3, tem-se $\lambda_{\max } \approx 3,008, C I \approx 0,004$ e $R I \approx 0,52$, resultando em $C R \approx 0,01$. Assim, com $C R \approx 0,10$, a matriz de comparações entre os critérios $\mathrm{X}, \mathrm{Y}$ e $\mathrm{Z}$ pode ser aceita.

No AHP original, as prioridades das alternativas com relação a cada critério, ou prioridades locais também são obtidas com autovetores de matrizes de comparações aos pares. Este é o modo de medição relativa (Salomon, 2016). No modo de medição absoluta, as alternativas são comparadas com padrões, ao invés de comparadas duas a duas. Uma vantagem da medicão absoluta é que não limita a análise a no máximo nove alternativas (Saaty e Ozdemir, 2003).

Dependendo do objetivo da análise deve-se optar pelo modo de síntese normal ou síntese ideal. No modo normal, as prioridades locais somam $100 \%$. Este modo é indicado, por exemplo, para casos de distribuição de recursos (Salomon et al., 2016). No modo de síntese ideal, a prioridade local da melhor alternativa é igual a 1, ou seja, 100\%; as demais prioridades serão porcentagens desta. A síntese ideal é indicada, por exemplo, para resolver problemas de seleção de fornecedores (Salomon et al., 2016). 


\section{Resultados}

Uma instituição federal de ensino tecnológico, na Região Norte do Brasil, no início de 2017, oferecia três cursos técnicos em um de seus campi localizados no interior do estado. Os cursos oferecidos eram agroindústria, agropecuária e aquicultura. A população atendida pela instituição era basicamente composta por moradores das zonas rurais de seis municípios situados $140 \mathrm{~km}$ ao sul da capital do estado.

Atendendo à demanda local e também aos anseios internos, a instituição decidiu ofertar um curso de nível superior. As cinco alternativas eram:

- BAG (Bacharelado em agronomia);

- BZO (Bacharelado em zootecnia);

- LCA (Licenciatura em ciências agrárias);

- TAG (Tecnologia em agroecologia);

- TAL (Tecnologia de alimentos).

O método AHP e o modelo BOCR foram apresentados para membros da administração da instituição. Na primeira reunião, quatro critérios de decisão foram identificados:

- Corpo docente;

- Infraestrutura;

- Produção local;

- Regulamentação.

No Modelo BOCR, os critérios B (Benefícios) e O (Oportunidades) relacionam-se com aspectos favoráveis, certos e incertos. A regulamentação de um curso é um benefício para a comunidade local (Costa Júnior, 2007) e para a instituição federal, uma organização pública. A produção local dos egressos do curso vai gerar oportunidades (Oliveira, 2013), relacionando-se com a cultura e restrições geográficas. Já os aspectos Corpo Docente e Infraestrutura relacionamse com aspectos desfavoráveis, tanto certos como incertos. A infraestrutura diz respeito às instalações físicas como salas de aula, laborat'orios, biblioteca, banheiros, quadras de esportes, etc. (Vieira e Albuquerque, 2001). Assim, a infraestrutura gera custos. O perfil do professor no novo ambiente de ensino é uma variável importante nas propostas pedagógicas (Perrenoud, 2000). Há, então, riscos de não atendimentos de expectativas. Portanto, há uma relação bionívoca entre os critérios identificados com os representantes da administração da instituição e os critérios do modelo BOCR:

- B (Regulamentação);

- O (Produção local);

- C (Infraestrutura);

- R (Corpo docente).

A Tabela 3 apresenta a matriz de comparações entre os critérios e o vetor de prioridades normalizado. As comparações foram obtidas por consenso, ouvindo-se dois representantes da administração da instituição em uma segunda reunião.

Tabela 3: Comparações e prioridades dos critérios B, O, C e R.

\begin{tabular}{cccccc}
\hline Critério & $\mathrm{R}$ & $\mathrm{C}$ & $\mathrm{O}$ & $\mathrm{B}$ & Prioridade \\
\hline $\mathrm{R}$ (Corpo docente) & 1 & 3 & $1 / 5$ & $1 / 5$ & $11 \%$ \\
$\mathrm{C}$ (Infraestrutura) & $1 / 3$ & 1 & $1 / 5$ & $1 / 5$ & $6 \%$ \\
O (Produção local) & 5 & 5 & 1 & $1 / 2$ & $34 \%$ \\
$\mathrm{C}$ (Regulamentação) & 5 & 5 & 2 & 1 & $49 \%$ \\
\hline
\end{tabular}

Fonte: Elaborada pelos autores.

Para a matriz de comparações apresentada na Tabela 3 , tem-se $\lambda_{\max } \approx 4,18, C I \approx 0,061 \mathrm{e}$ $R I=0,90$, resultando em $C R \approx 0,068$. Assim, com um $C R \leq 0,10$, a matriz de comparações entre os critérios $\mathrm{B}, \mathrm{O}, \mathrm{C}$ e $\mathrm{R}$ pode ser aceita. 
A Tabela 4 apresenta a matriz de comparações entre as alternativas com relação ao corpo docente. $\mathrm{O}$ vetor de prioridades foi normalizado utilizando-se o modo de síntese ideal. As comparações foram obtidas por consenso, na mesma segunda reunião.

Tabela 4: Comparações e prioridades das alternativas com relação ao corpo docente.

\begin{tabular}{ccccccc}
\hline Curso & BAG & BZO & LCA & TAG & TAL & Prioridade \\
\hline BAG (Bacharelado em agronomia) & 1 & $1 / 2$ & $1 / 3$ & 3 & 3 & $53 \%$ \\
BZO (Bacharelado em zootecnia) & 2 & 1 & 2 & 3 & 3 & $100 \%$ \\
LCA (Licenciatura em ciências agrárias) & 3 & $1 / 2$ & 1 & 5 & 3 & $91 \%$ \\
TAG (Tecnologia em agroecologia) & $1 / 3$ & $1 / 3$ & $1 / 5$ & 1 & 2 & $26 \%$ \\
TAL (Tecnologia de alimentos) & $1 / 3$ & $1 / 3$ & $1 / 3$ & $1 / 2$ & 1 & $22 \%$ \\
\hline
\end{tabular}

Fonte: Elaborada pelos autores.

Para a matriz de comparações apresentada na Tabela 4 , tem-se $\lambda_{\max } \approx 5,33, C R \approx 0,085$ e $R I=1,12$, resultando em $C I \approx 0,075$. Assim, com um $C R \leq 0,10$, a matriz de comparações entre as alternativas com relação ao corpo docente pode ser aceita.

Comparações aos pares entre as alternativas para os demais critérios também foram obtidas, por consenso, com os representantes da administração da instituição. Todas as matrizes de comparações apresentam $C R \leq 0,10$.

A Tabela 5 apresenta a matriz de decisão, com as prioridades locais para cada critério, e o vetor de decisão com as prioridades globais. Nota-se que o curso de Bacharelado em Zootecnia obteve a máxima prioridade em todos os critérios e, portanto, também obteve prioridade global máxima. Este fato não estava explicitamente reconhecido pelos administradores da instituição antes da aplicação do método AHP.

Tabela 5: Matriz e vetor de decisão.

\begin{tabular}{cccccc}
\hline Critério & $\mathrm{R}$ & $\mathrm{C}$ & $\mathrm{O}$ & $\mathrm{B}$ & Global \\
\hline Bacharelado em agronomia & $53 \%$ & $49 \%$ & $58 \%$ & $49 \%$ & $53 \%$ \\
Bacharelado em zootecnia & $100 \%$ & $100 \%$ & $100 \%$ & $100 \%$ & $100 \%$ \\
Licenciatura em ciências agrárias & $91 \%$ & $77 \%$ & $74 \%$ & $84 \%$ & $81 \%$ \\
Tecnologia de alimentos & $22 \%$ & $20 \%$ & $15 \%$ & $21 \%$ & $19 \%$ \\
Tecnologia em agroecologia & $26 \%$ & $24 \%$ & $29 \%$ & $31 \%$ & $30 \%$ \\
\hline
\end{tabular}

Fonte: Elaborada pelos autores.

\section{Conclusões}

A aplicação do método AHP gerou um resultado conclusivo: Bacharelado em Zootecnia é o curso de nível superior indicado para a instituição estudada. Assim, a aplicação do método AHP pode ser considerada eficaz, uma vez que apenas um curso foi indicado. Em se tratando de uma instituição de ensino profissional, na Região Norte do país, a priori, a implantação de qualquer curso seria bem-vinda. Assim, não se tinha noção de que o curso de Bacharelado em Zootecnia era tão superior que os demais cursos. Esta escolha ficou evidente apenas após a aplicação do método AHP.

A aplicação também pode ser considerada eficiente, pois, foi realizada em apenas duas reuniões com os representantes da instituição. Além disso, nenhum software específico foi necessário: o AHP foi aplicado apenas com planilhas eletrônicas.

Duas propostas para pesquisas futuras surgem dos resultados encontrados:

- Testar o modelo de análise multicritério, com os mesmos critérios, em outra instituição. Assim, os resultados podem ser comparativamente analisados.

- Aplicar um método diferente do AHP, ou combinar teorias como a teoria dos conjuntos nebulosos (fuzzy sets). 
Agradecimentos. Esta pesquisa contou com apoio financeiro da Fundação de Amparo à Pesquisa do Estado de São Paulo (FAPESP), Processo no 2017/22963-6. Os autores agradecem aos avaliadores anônimos por sua inestimável contribuição.

\section{Referências}

Costa, J. J., Lima, T. J. B., Meza, E. B. M. e Tammela, I. Uso do método ahp clássico para auxiliar na escolha de um novo curso superior em um campus da UFF no interior. In: Anais do $X$ Congresso Nacional de Excelência em Gestão, Rio de Janeiro. UFF, 2014. p. 1-12.

Costa Júnior, H. P. Estudo da participação e permanência dos jovens na agricultura familiar na localidade do Ancorado em Rosário da Limeira - MG. 131p. Dissertação (Mestrado Profissional em Meio Ambiente e Sustentabilidade) - Centro Universitário de Caratinga, Caratinga-MG, 2007.

Ishizaka, A. e Nemery, P. Multi-criteria decision analysis. Methods and software. Chichester, UK: Wiley, 2013.

Khan, S. A., Chaabane, A. e Dweiri, F. T. Multi-criteria decision-making methods application in supply chain management: A systematic literature review. In: Salomon, V. A. P., (e.), Multicriteria methods and techniques applied to supply chain management, cap. 1, p. 3-31. London: InTechOpen, 2018.

$\mathrm{Mu}$, E. What constitutes a novel AHP/ANP study? International Journal of the Analytic Hierarchy Process, v. 9, n. 1, p. 1, 2017.

Oliveira, A. J. Programa REUNI nas Instituições de ensino superior federal (IFES) brasileiras: um estudo da eficiência operacional por meio da análise envoltória de dados (DEA) no período de 2006 a 2012. 146p. Dissertação (Mestrado em Contabilidade) - Setor de Ciências Sociais Aplicadas, Universidade Federal do Paraná, Curitiba-PR, 2013.

Perrenoud, P. Dez novas competências para ensinar. Porto Alegre: Artmed Editora, 2000.

Ranieri, N. Autonomia universitária e lei de responsabilidade fiscal: relações e implicações. Revista USP, n. 105, p. 33-42, 2015.

Roy, B. The optimisation problem formulation: Criticism and overstepping. Journal of the Operational Research Society, v. 32, n. 6, p. 427-436, 1981.

Saaty, T. L. The analytic hierarchy process. New York: McGraw-Hill, 1980.

Saaty, T. L. Mathematical principles of decision making. Pittsburgh, USA: RWS Publications, 2015.

Saaty, T. L. e Islam, R. Hierarchon Volume II: A dictionary of AHP hierarchies. Pittsburgh, USA: RWS Publications, 2015.

Saaty, T. L. e Ozdemir, M. S. Why the magic number seven plus or minus two. Mathematical and Computer Modelling, v. 38, n. 3-4, p. 233-244, 2003.

Salomon, V. A. P. Absolute measurement and ideal synthesis on AHP. International Journal of the Analytic Hierarchy Process, v. 8, n. 3, p. 538-545, 2016.

Salomon, V. A. P., Tramarico, C. L. e Marins, F. A. S. Analytic hierarchy process applied to supply chain management. In: DeFelice, F., Saaty, T. L. e Petrillo, A. (ee.), Applications and theory of analytic hierarchy process - Decision making for strategic decisions, cap. 1, p. 1-16. London: InTechOpen, 2016.

Salomon, V. A. P., Zinoviev, A. e Zinovieva, O. Book review: Alessio Ishizaka and Philippe Nemery's multi-criteria decision analysis. International Journal of the Analytic Hierarchy Process, v. 5, n. 2, p. 284-287, 2013. 
Silva, A. F., Marins, F. A. S. e Santos, M. V. B. Programação por metas, análise por envoltória de dados e teoria fuzzy na avaliação da eficiência sob incerteza: Aplicação em minifábricas do segmento de autopeças. Gestão \& Produção, v. 21, n. 3, p. 543-554, 2014.

Steuer, R. E., Zionts, S., Koksalan, M., Miettinen, K. e Wallenius, J. Short MCDM history, 2017. Disponível em: http://www.mcdmsociety.org/content/short-mcdm-history-0. Acesso em: 16/10/2018.

Tramarico, C. L., Salomon, V. A. P., Marins, F. A. S. e Muniz Jr., J. Modelagem com AHP e BOCR para a seleção de prestadores de serviços logísticos. Pesquisa Operacional para o Desenvolvimento, v. 4, n. 2, p. 139-159, 2012.

Vieira, S. L. e Albuquerque, M. G. M. Estrutura e funcionamento de educação básica. Fortaleza: Edições Demócrito Rocha, UECE, 2001.

Wallenius, J., Dyer, J. S., Fishburn, P. C., Steuer, R. E., Zionts, S. e Deb, K. Multiple criteria decision making, multiattribute utility theory: Recent accomplishments and what lies ahead. Management Science, v. 54, n. 7, p. 1336-1349, 2008.

Williams, D. IMRAD content structure for research papers, literary reviews, and abstracts for science writing. CUNY Academic Works, City University of New York, 2018.

Zopounidis, C. e Pardalos, P. M. (ee.). Handbook of multicriteria analysis. Berlin: Springer, 2010. 